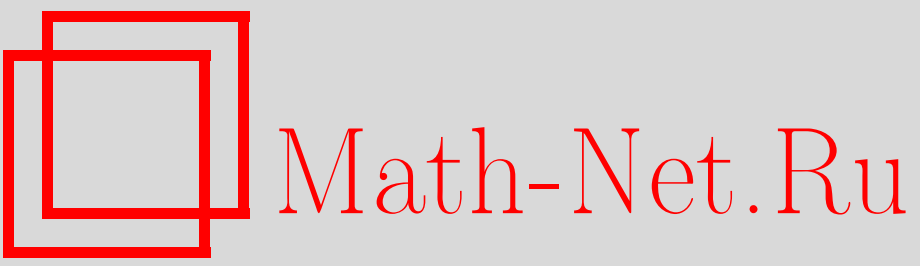

А. Д. Баев, А. А. Бабайцев, В. Д. Харченко, Теорема об ограниченности одного класса псевдодифференциальных уравнений с вырождением, Итоги науки и техн. Сер. Соврем. мат. и ее прил. Темат. обз., 2021, том 193, 3-10

DOI: https://doi.org/10.36535/0233-6723-2021-193-3-10

Использование Общероссийского математического портала Math-Net.Ru подразумевает, что вы прочитали и согласны с пользовательским соглашением

http://www.mathnet.ru/rus/agreement

Параметры загрузки:

IP : 54.237 .206 .68

26 апреля 2023 г., 13:04:45 


\title{
ТЕОРЕМА ОБ ОГРАНИЧЕННОСТИ ОДНОГО КЛАССА ПСЕВДОДИФФЕРЕНЦИАЛЬНЫХ УРАВНЕНИЙ С ВЫРОЖДДЕНИЕМ
}

\author{
(c) 2021 г. $\quad$ А. Д. БАЕВ, А. А. БАБАЙЦЕВ, В. Д. ХАРЧЕНКО
}

\begin{abstract}
АннотАция. Статья посвящена доказательству теоремы об ограниченности для одного класса псевдодифференциальных уравнений с вырождением. Рассматривается новый класс переменных символов, зависящих также от комплексного параметра. Псевдодифференциальные операторы построены по специальному интегральному преобразованию. Теорема об ограниченности таких операторов доказывается в специальных весовых пространствах типа пространств С. Л. Соболева.
\end{abstract}

Ключевые слова: псевдодифференциальный оператор с вырождением, теорема об ограниченности, весовое пространство Соболева.

\section{BOUNDEDNESS THEOREM FOR ONE CLASS OF PSEUDODIFFERENTIAL EQUATIONS WITH DEGENERATION}

\author{
(c) 2021 A. D. BAEV, A. A. BABAITSEV, V. D. KHARCHENKO
}

\begin{abstract}
In this paper, we prove a boundedness theorem for one class of pseudodifferential equations with degeneration. We consider a new class of variable symbols depending on a complex parameter. Pseudodifferential operators are constructed by a special integral transformation. The boundedness theorem for these operators is proved in special weighted Sobolev-type spaces.
\end{abstract}

Keywords and phrases: pseudodifferential operator with degeneration, boundedness theorem, weighted Sobolev space.

AMS Subject Classification: 35S05

1. Введение. Вырождающиеся дифференциальные уравнения используются при моделировании различных физических процессов, в которых граница области оказывает существенное влияние на процессы, происходящие вблизи границы. В этом случае на границе области может меняться как тип уравнений, так и их порядок. Такие уравнения используются при исследовании стационарных процессов конвекции - диффузии в неоднородных анизотропных средах, характерных тем, что при приближении к границе коэффициент диффузии стремится к нулю. В частности, к таким уравнениям приводит математическое моделирование процессов фильтрации идеального баротропного газа в неоднородной анизотропной пористой среде процессов фильтрации двухфазных жидкостей, в том числе, процессов вытеснения нефти водой из пористой среды. Подобные уравнения возникают при моделировании процесса распространения примеси в жидкокристаллическом растворе, находящемся во внешнем электрическом поле, при исследовании

Работа выполнена при поддержке Министерства образования и науки РФ (проект № 14.Z50.31.0037) и Российского научного фонда (проект № 19.11.00197). 
стационарной задачи о контакте мягкой оболочки с препятствием, при расчете линейных стационарных магнитных осесимметричных полей в неоднородных анизотропных средах. Такие уравнения являются также обобщением сингулярно возмущенных уравнений конвекции - диффузии. Кроме того, известно, что нахождение решения краевой задачи для эллиптического уравнения эквивалентно минимизации некоторого функционала. В теории управления задача о минимуме некоторого функционала соответствует задаче об оптимальном управлении. Вырождающимся эллиптическим уравнениям соответствуют вырожденные или особые оптимальные управления.

Краевые задачи для вырождающихся уравнений относятся к «неклассическим» задачам математической физики. Основная трудность, возникающая в теории вырождающихся эллиптических уравнений, связана с влиянием младших (в смысле теории регулярных эллиптических операторов) членов уравнения на постановку граничных задач и их коэрцитивную разрешимость.

Вырождающиеся эллиптические уравнения второго порядка и граничные задачи для них достаточно хорошо изучены. Фундаментальные результаты в этом направлении принадлежат М. В. Келдышу [15]. Полученные им результаты затем развивались и обобщались О. А. Олейник [18]. Обобщенные решения вырождающихся эллиптических уравнений второго порядка впервые были рассмотрены в работах С. Г. Михлина [17] и М. И. Вишика [12]. Исследование вырождающихся эллиптических уравнений высокого порядка (при «степенном» характере вырождения) было начато в работах М. И. Вишика и В. В. Грушина [13]. Затем ряд результатов для некоторых классов вырождающихся уравнений высокого порядка был получен В. П. Глушко [14], С. З. Левендорским [16], А. Д. Баевым, Р. А. Ковалевским, А. А. Бабайцевым $[1-5,7,9,10]$.

Настоящая работа посвящена доказательству теоремы об ограниченности одного класса вырождающихся псевдодифференциальных операторов с переменным символом, зависящим также от комплексного параметра.

В работе систематически используется специальное интегральное преобразование $F_{\alpha}$, введенное в [1]. Преобразование $F_{\alpha}$ позволяет ввести в рассмотрение специальный класс весовых псевдодифференциальных операторов. Весовые псевдодифференциальные операторы с постоянным по $y$ символом были изучены в [1], а в $[6,8,11]$ были исследованы некоторые классы весовых псевдодифференциальных операторов с переменным символом.

В работе исследуются весовые псевдодифференциальные операторы с переменным по $t$ символом, зависящим от комплексного параметра, из класса $S_{\alpha, p}^{\sigma}, p \in Q=\left\{p \in \mathbb{C},|\arg p|<\frac{\pi}{2},|p|>0\right\}$. Доказывается теорема об ограниченности таких операторов в специальных весовых пространствах типа пространств С. Л. Соболева.

Рассмотрим функцию $\alpha(t), t \in \mathbb{R}_{+}^{1}$, для которой выполняются условия: $\alpha(+0)=\alpha^{\prime}(+0)=0$, $\alpha(t)>0$ при $t>0, \alpha(t)=$ const для $t \geqslant d$ при некотором $d>0$.

Рассмотрим интегральное преобразование

$$
F_{\alpha}[u(t)](\eta)=\int_{0}^{+\infty} u(t) \exp \left[i \eta \int_{t}^{d} \frac{d \rho}{\alpha(\rho)}\right] \frac{d t}{\sqrt{\alpha(t)}}
$$

которое определено первоначально на функциях $u(t) \in C_{0}^{\infty}\left(\mathbb{R}_{+}^{1}\right)$. Здесь $C_{0}^{\infty}\left(\mathbb{R}_{+}^{1}\right)$ - пространство бесконечно дифференцируемых финитных функций, носитель которых принадлежит $\mathbb{R}_{+}^{1}$. Преобразование (1) и преобразование Фурье

$$
F_{\tau \rightarrow \eta}[u]=\int_{-\infty}^{+\infty} u(\tau) \exp (i \eta \tau) d \tau, \quad \eta \in \mathbb{R}^{1}
$$

связаны следующим соотношением

$$
F_{\alpha}[u(t)](\eta)=F_{\tau \rightarrow \eta}\left[u_{\alpha}(\tau)\right]
$$


где $u_{\alpha}(\tau)=\left.\sqrt{\alpha(t)} u(t)\right|_{t=\varphi^{-1}(\tau)}, t=\varphi^{-1}(\tau)$ - функция, обратная к функции

$$
\tau=\varphi(t)=\int_{t}^{d} \frac{d \rho}{\alpha(\rho)} .
$$

Для преобразования $F_{\alpha}$ справедлив аналог равенства Парсеваля

$$
\left\|F_{\alpha}[u](\eta)\right\|_{L_{2}\left(\mathbb{R}^{1}\right)}=\sqrt{2 \pi}\|u\|_{L_{2}\left(\mathbb{R}_{+}^{1}\right)} .
$$

Равенство (3) дает возможность расширить преобразование (1) до непрерывного преобразования, осуществляющего гомеоморфизм пространств $L_{2}\left(\mathbb{R}^{1}\right)$ и $L_{2}\left(\mathbb{R}_{+}^{1}\right)$, а также рассмотреть преобразование $F_{\alpha}$ на некоторых классах обобщенных функций. Для расширенного таким образом преобразования $F_{\alpha}$ сохраним старое обозначение. Обозначим через $F_{\alpha}^{-1}$ обратное к $F_{\alpha}$ преобразование. Это преобразование можно записать в виде

$$
F_{\alpha}^{-1}[w(\eta)](t)=\left.\frac{1}{\sqrt{\alpha(t)}} F_{\eta \rightarrow \tau}^{-1}[w(\eta)]\right|_{\tau=\varphi(t)} .
$$

Можно показать, что для функции $u(t) \in C_{0}^{\infty}\left(\overline{\mathbb{R}}_{+}^{1}\right)$ справедливы равенства

$$
F_{\alpha}\left[D_{\alpha, t}^{j} u\right](\eta)=\eta^{j} F_{\alpha}[u](\eta), \quad j=1,2, \ldots,
$$

где

$$
D_{\alpha, t}=\frac{1}{i} \sqrt{\alpha(t)} \partial_{t} \sqrt{\alpha(t)} \quad \partial_{t}=\frac{\partial}{\partial t} .
$$

Определим пространства $H_{s, \alpha}\left(\mathbb{R}_{+}^{n}\right), H_{s, \alpha, q}\left(\mathbb{R}_{+}^{n}\right)$ следующим образом.

Определение 1. Пространство $H_{s, \alpha}\left(\mathbb{R}_{+}^{n}\right)(s$ - действительное число) состоит из всех функций пространства $L_{2}\left(\mathbb{R}_{+}^{n}\right)$, для которых конечна норма

$$
\|v,|p|\|_{s, \alpha}^{2}=\int_{\mathbb{R}^{n}}\left(|p|^{2}+|\xi|^{2}+\eta^{2}\right)^{s}\left|F_{\alpha} F_{x \rightarrow \xi}[v(x, y)]\right|^{2} d \xi d \eta
$$

зависящая от комплексного параметра $p \in Q=\{p \in \mathbb{C},|\arg p|<\pi / 2,|p|>0\}$.

Определение 2. Пространство $H_{s, \alpha, q}\left(\mathbb{R}_{+}^{n}\right)(s \geqslant 0, q>1)$ состоит из всех функций $v(x, y) \in$ $H_{s, \alpha}\left(\mathbb{R}_{+}^{n}\right)$, для которых конечна норма

$$
\|v,|p|\|_{s, \alpha, q}=\left\{\sum_{l=0}^{[s / q]}\left\|F_{\xi \rightarrow x}^{-1} F_{\alpha}^{-1}\left[\left(|p|^{2}+|\xi|^{2}+\eta^{2}\right)^{(s-q l) / 2} F_{\alpha} F_{x \rightarrow \xi}\left[\partial_{y}^{l} v\right]\right]\right\|_{L_{2}\left(\mathbb{R}_{+}^{n}\right)}^{2}\right\}^{1 / 2},
$$

зависящая от комплексного параметра. Здесь $[s / q]$ - целая часть числа $s / q$.

Пусть выполнено следующее условие.

Условие 1. Существует такое число $\nu \in(0,1]$, что

$$
\left|\alpha^{\prime}(t) \alpha^{-\nu}(t)\right| \leqslant c<\infty, \quad t \in[0,+\infty) .
$$

Кроме того, $\alpha(y) \in C^{s_{1}}[0,+\infty)$ для некоторого $s_{1} \geqslant 2 N-|\sigma|$, где

$$
N \geqslant \max _{0 \leqslant p_{1} \leqslant l}\left\{2 p_{1}+\frac{l-p_{1}+3 / 2}{\nu}+1, \sigma+1, \sigma+\frac{l}{2}\right\}, \quad l=1,2, \ldots,
$$

$\sigma$ - некоторое действительное число.

Можно показать, что указанное выше число $\nu$ существует, если $\alpha(+0)=\alpha^{\prime}(+0)=0$.

С помощью преобразования (1) и преобразования Фурье $F_{x \rightarrow \xi}=F_{x_{1} \rightarrow \xi_{1}} F_{x_{2} \rightarrow \xi_{2}} \ldots F_{x_{n-1} \rightarrow \xi_{n-1}}$ определим весовой псевдодифференциальный оператор по формуле

$$
G^{(\sigma)}\left(p, t, D_{x}, D_{\alpha, t}\right) v(x, t)=F_{\alpha}^{-1} F_{\xi \rightarrow x}^{-1}\left[g(p, t, \xi, \eta) F_{x \rightarrow \xi} F_{\alpha}[v(x, t)]\right] .
$$


Определение 3. Будем говорить, что символ $g(p, t, \xi, \eta)$ весового псевдодифференциального оператора $G^{(\sigma)}\left(p, t, D_{x}, D_{\alpha, y}\right)$ принадлежит классу символов $S_{\alpha, \rho}^{\sigma, p}(\Omega)$, где $\Omega \subset \overline{\mathbb{R}}_{+}^{1}, \sigma \in \mathbb{R}^{1}, p \in Q=$ $\{p \in \mathbb{C},|\arg p|<\pi / 2,|p|>0\}$, если функция $g(p, t, \xi, \eta)$ является бесконечно дифференцируемой функцией по переменной $t \in \Omega$ и по переменной $\eta \in \mathbb{R}^{1}$, причем при всех $j=0,1,2, \ldots, l=$ $0,1,2, \ldots$ справедливы оценки

$$
\left|\left(\alpha(t) \partial_{t}\right)^{j} \partial_{\eta}^{l} \lambda(p, t, \xi, \eta)\right| \leqslant c_{j l}\left(|p|^{2}+|\xi|+|\eta|\right)^{\sigma-\rho l}
$$

с константами $c_{j l}>0$, не зависящими от $p \in Q, \xi \in \mathbb{R}^{n-1}, \eta \in \mathbb{R}^{1}, \rho \in(0 ; 1], t \in K$, где $K \subset \Omega-$ произвольный отрезок. Здесь $\sigma$-действительное число.

Доказаны следующие утверждения.

Теорема 1. Пусть $g(p, t, \xi, \eta) \in S_{\alpha, \rho}^{m, p}(\Omega), m$-действительное число, $p \in Q=\{p \in \mathbb{C}$, $|\arg p|<\pi / 2,|p|>0\}$. Тогда весовой псевдодифференииальный оператор $G\left(p, t, D_{x}, D_{\alpha, t}\right)$ для любого действительного s есть ограниченный оператор из $H_{s+m, \alpha}\left(\mathbb{R}_{+}^{n}\right)$ в $H_{s, \alpha}\left(\mathbb{R}_{+}^{n}\right)$.

2. Схема доказательства теоремы 1. Заметим, что

$$
\begin{aligned}
\left\|G\left(p, t, \xi, D_{\alpha, t}\right) u(t)\right\|_{L_{2}\left(\mathbb{R}_{+}^{1}\right)}^{2}=\int_{0}^{\infty}\left|F_{\alpha}^{-1}\left[g(p, t, \xi, \eta) F_{\alpha}[u]\right]\right|^{2} d t= \\
\quad=\left.\int_{0}^{\infty}\left|\frac{1}{\sqrt{\alpha(t)}} F_{\eta \rightarrow \tau}^{-1}\left[g\left(p, \varphi^{-1}(\tau), \xi, \eta\right) F_{\tau \rightarrow \eta}\left[u_{\alpha}(\tau)\right]\right]\right|^{2}\right|_{\tau=\varphi(t)} d t .
\end{aligned}
$$

Сделав замену переменной $t=\varphi^{-1}(\tau)$, получим $d t=\left(\varphi^{-1}(\tau)\right)_{\tau}^{\prime} d \tau=-\left.\alpha(t)\right|_{t=\varphi^{-1}(\tau)} d \tau$. Таким образом, получаем равенство

$$
\begin{aligned}
& \| G\left(p, t, \xi, D_{\alpha, t}\right) u(t)\left\|_{L_{2}\left(\mathbb{R}_{+}^{1}\right)}^{2}=\int_{-\infty}^{\infty} \mid F_{\eta \rightarrow \tau}^{-1}\left[g\left(p, \varphi^{-1}(\tau), \xi, \eta\right) F_{\tau \rightarrow \eta}\left[u_{\alpha}(\tau)\right]\right]\right\| \|^{2} d \tau= \\
&=\left\|F_{\eta \rightarrow \tau}^{-1}\left[g\left(p, \varphi^{-1}(\tau), \xi, \eta\right) F_{\tau \rightarrow \eta}\left[u_{\alpha}(\tau)\right]\right]\right\|_{L_{2}\left(\mathbb{R}^{1}\right)}^{2}=\left\|G\left(p, \tau, \xi, D_{\tau}\right) u_{\alpha}(\tau)\right\|_{L_{2}\left(\mathbb{R}^{1}\right)}^{2}
\end{aligned}
$$

Следовательно, норма оператора $G\left(p, t, \xi, D_{\alpha, t}\right)$ в пространстве $L_{2}\left(\mathbb{R}_{+}^{1}\right)$ равна норме оператора $G\left(p, \tau, \xi, D_{\tau}\right)$, определенного в пространстве $L_{2}\left(\mathbb{R}^{1}\right)$.

Справедливо следующее утверждение.

Теорема 2. Пусть $g(p, t, \xi, \eta) \in S_{\alpha, \rho}^{0, p}(\Omega), \Omega \subset \overline{\mathbb{R}}_{+}^{1}, p \in Q=\{p \in \mathbb{C},|\arg p|<\pi / 2,|p|>0\}$. Тогда существует такал константа с $>0$, что для любой функиии $u(t) \in C_{0}^{\infty}\left(\mathbb{R}_{+}^{1}\right)$ справедлива оченка

$$
\left\|G\left(p, t, \xi, D_{\alpha, t}\right) u\right\|_{L_{2}\left(\mathbb{R}_{+}^{1}\right)} \leqslant c\|u\|_{L_{2}\left(\mathbb{R}_{+}^{1}\right)}
$$

с константой $c>0$, не зависящей от параметра $p$.

Доказательство. Докажем вначале теорему 2 при следующем дополнительном предположении. Будем считать, что $g\left(p, \varphi^{-1}(\tau), \xi, \eta\right)=0$ при $|\tau-a|>A$, где $A>0$ - некоторое число, $a \in \mathbb{R}^{1}$.

Рассмотрим равенство

$$
f(\tau)=\int_{-\infty}^{\infty} e^{-i \tau \eta} p\left(\varphi^{-1}(\tau), \xi, \eta\right) F_{\tau \rightarrow \eta}\left[u_{\alpha}(\tau)\right] d \eta .
$$

Перейдя в этом равенстве к преобразованию Фурье, получим

$$
\tilde{f}(z)=\int_{-\infty}^{\infty} \tilde{g}_{1}(p, z-\eta, \xi, \eta) \tilde{u}_{\alpha}(\eta) d \eta
$$


где

$$
g_{1}(p, \tau, \xi, \eta)=g\left(p, \varphi^{-1}(\tau), \xi, \eta\right), \quad \tilde{g}_{1}(p, z, \xi, \eta)=F_{\tau \rightarrow z}\left[g_{1}(p, \tau, \xi, \eta)\right], \quad \tilde{u}_{\alpha}(\eta)=F_{\tau \rightarrow \eta}\left[u_{\alpha}(\tau)\right]
$$

С помощью интегрирования по частям в интеграле

$$
\tilde{g}_{1}(p, z, \xi, \eta)=\int_{-\infty}^{\infty} e^{i \tau z} g_{1}(p, \tau, \xi, \eta) d \tau
$$

получим, что с некоторыми константами $c>0$ выполняются неравенства

$$
\int_{-\infty}^{\infty}\left|\tilde{g}_{1}(p, z-\eta, \xi, \eta)\right| d \eta \leqslant c \int \frac{d \eta}{(|p|+|\eta|)^{2}}, \quad \int_{-\infty}^{\infty}\left|\tilde{g}_{1}(p, z-\eta, \xi, \eta)\right| d z \leqslant c \int \frac{d z}{(|p|+|z|)^{2}}
$$

Отсюда и из (10) выводим неравенство

$$
\|\tilde{f}(z)\|_{L_{2}\left(\mathbb{R}^{1}\right)} \leqslant c\left\|u_{\alpha}(\tau)\right\|_{L_{2}\left(\mathbb{R}^{1}\right)} .
$$

Заметив, что

$$
\left\|u_{\alpha}(\tau)\right\|_{L_{2}\left(\mathbb{R}^{1}\right)}=\|u(t)\|_{L_{2}\left(\mathbb{R}_{+}^{1}\right)},
$$

получаем из (11) утверждение теоремы 2 при дополнительном предположении.

Докажем теперь теорему 2 в общем случае. Выберем некоторое $A>0$ и установим, что для любого $a \in \mathbb{R}^{1}$ справедливо неравенство

$$
\begin{aligned}
& \int_{|\tau-a| \leqslant A}\left|G\left(p, \tau, \xi, D_{\tau}\right) u_{\alpha}(\tau)\right|^{2} d \tau \leqslant \\
& \leqslant c\left\{\int_{|\tau-a| \leqslant 3 A}\left|u_{\alpha}(\tau)\right|^{2} d \tau+\int_{|\tau-a| \leqslant A}\left(\int_{-\infty}^{\infty} r(p, \tau-y)|u(y)| d y\right)^{2} d \tau\right\}
\end{aligned}
$$

где $r(p, z)=(|p|+|z|)^{-2}$. Константа $c$ не зависит от выбора функции $u(t) \in C_{0}^{\infty}\left(\mathbb{R}_{+}^{1}\right)$ и $a \in \mathbb{R}^{1}$. Пусть $\varphi(\tau)$ - такая основная функция с носителем в шаре $|\tau| \leqslant 3 A$, что $\varphi(\tau)=1$ в шаре $|\tau| \leqslant 2 A$, причем $|\varphi(\tau)| \leqslant 1$. Тогда для оператора $\varphi(\tau-a) P\left(\tau, \xi, D_{\tau}\right)$ символ равен нулю при $|\tau-a|>3 A$ и мы можем воспользоваться доказанной только что оценкой:

$$
\left\|\varphi(\tau-a) G\left(p, \tau, \xi, D_{\tau}\right) v_{\alpha}(\tau)\right\|_{L_{2}\left(\mathbb{R}^{1}\right)} \leqslant c\left\|v_{\alpha}(\tau)\right\|_{L_{2}\left(\mathbb{R}^{1}\right)}=c\|v(t)\|_{L_{2}\left(\mathbb{R}_{+}^{1}\right)}
$$

причем из предыдущих рассуждений видно, что константа $c>0$ не зависит от $v(t) \in C_{0}^{\infty}\left(\mathbb{R}_{+}^{1}\right)$ и $a \in \mathbb{R}^{1}$.

Обозначим $f(p, \tau)=G\left(p, \tau, \xi, D_{\tau}\right) u_{\alpha}(\tau)$ и заметим, что при $|\tau-a| \leqslant 2 A$ справедливо равенство

$$
f(p, \tau)=\varphi(\tau-a) G\left(p, \tau, \xi, D_{\tau}\right)\left[\varphi(\tau-a) u_{\alpha}(\tau)\right]+G\left(p, \tau, \xi, D_{\tau}\right)\left[(1-\varphi(\tau-a)) u_{\alpha}(\tau)\right] .
$$

Воспользовавшись неравенством (14), получим оценку

следовательно,

$$
\left\|\varphi(\tau-a) G\left(p, \tau, \xi, D_{\tau}\right)\left[\varphi(\tau-a) u_{\alpha}(\tau)\right]\right\|_{L_{2}\left(\mathbb{R}^{1}\right)} \leqslant c\left\|\varphi(\tau-a) u_{\alpha}(\tau)\right\|_{L_{2}\left(\mathbb{R}^{1}\right)}
$$

$$
\int_{|\tau-a| \leqslant A}\left|g\left(p, \tau, \xi, D_{\tau}\right)\left[\varphi(\tau-a) u_{\alpha}(\tau)\right]\right|^{2} d \tau \leqslant c \int_{|\tau-a| \leqslant 3 A}\left|u_{\alpha}(\tau)\right|^{2} d \tau .
$$

Так как $1-\varphi(\tau-a)=0$ в шаре $|\tau-a| \leqslant 2 A$, то

$$
G\left(p, \tau, \xi, D_{\tau}\right)\left[(1-\varphi(\tau-a)) u_{\alpha}(\tau)\right]=\int_{|y-a|>2 A} k(p, \tau, \xi, \tau-y)(1-\varphi(y-a)) u_{\alpha}(y) d y
$$

где $k(p, \tau, \xi, z)$ - ядро псевдодифференциального оператора $G\left(p, \tau, \xi, D_{\tau}\right)$. 
Заметим, что

$$
\left|\partial_{\tau}^{i} \partial_{\eta}^{l} g\left(p, \varphi^{-1}(\tau), \xi, \eta\right)\right| \leqslant c_{i l}(|p|+|\xi|+|\eta|)^{m-\rho l} .
$$

По определению ядра имеем

$$
k(p, \tau, \xi, z)=F_{\eta \rightarrow z}^{-1}\left[g\left(p, \varphi^{-1}(\tau), \xi, \eta\right)\right] .
$$

Так как преобразование Фурье любой абсолютно интегрируемой функции является непрерывной и ограниченной функцией, то можно утверждать, что функция $z^{q} \partial_{\tau}^{i} \partial_{z}^{j} k(p, \tau, \xi, z)$ для любых $i, j$ при $q>m+j+1$ будет непрерывной и ограниченной функцией при всех $\tau \in \mathbb{R}^{1}, z \in \mathbb{R}^{1}$ и

$$
|z|^{q}\left|\partial_{\tau}^{i} \partial_{z}^{j} k(p, \tau, \xi, z)\right| \leqslant c_{i j}<\infty
$$

т.е. при всех $z \neq 0$ справедливо неравенство

$$
\left|\partial_{\tau}^{i} \partial_{z}^{j} k(p, \tau, \xi, z)\right| \leqslant c_{i j}|z|^{-q} .
$$

Из (16) получим, что

$$
|k(p, \tau, \xi, \tau-y)| \leqslant c(|p|+|\tau-y|)^{-2} \text { при }|\tau-y| \geqslant A .
$$

Отсюда при $|\tau-a| \geqslant A$ получаем, что

$$
\left|G\left(p, \tau, \xi, D_{\tau}\right)\left[(1-\varphi(\tau-a)) u_{\alpha}(\tau)\right]\right| \leqslant c \int_{-\infty}^{\infty}(|p|+|\tau-y|)^{-2}|u(y)| d y
$$

Неравенство (13) вытекает теперь из неравенств (15) и (17).

Чтобы закончить доказательство теоремы, достаточно в неравенстве (13) выбрать $A=1$ и просуммировать неравенства (13) по всем $a$, принадлежащим множеству целых чисел. Так как отрезки $|\tau-a| \leqslant 1$ покрывают все $\mathbb{R}^{1}$, причем каждая точка $\tau \in \mathbb{R}^{1}$ принадлежит не более чем двум таким отрезкам, то получим, что с некоторой константой выполняется неравенство

$$
\int_{-\infty}^{\infty}\left|G\left(p, \tau, \xi, D_{\tau}\right) u_{\alpha}(\tau)\right|^{2} d \tau \leqslant c\left\{\int_{-\infty}^{\infty}\left|u_{\alpha}(\tau)\right|^{2} d \tau+\int_{-\infty}^{\infty}\left(\int_{-\infty}^{\infty}(|p|+|\tau-y|)^{-2}\left|u_{\alpha}(y)\right| d y\right)^{2} d \tau\right\} .
$$

Остается заметить, что

$$
\int_{-\infty}^{\infty}\left(\int_{-\infty}^{\infty}(|p|+|\tau-y|)^{-2}\left|u_{\alpha}(y)\right| d y\right)^{2} d \tau \leqslant c \int_{-\infty}^{\infty}\left|u_{\alpha}(\tau)\right|^{2} d \tau=c \int_{0}^{\infty}|u(t)|^{2} d t .
$$

Теорема 3. Пусть $g(p, t, \xi, \eta) \in S_{\alpha, p}^{m}(\Omega)(m \in \mathbb{R}), \Omega \subset \overline{\mathbb{R}}_{+}^{1}, p \in Q=\{p \in \mathbb{C},|\arg p|<\pi / 2$, $|p|>0\}$. Тогда весовой псевдодифберенииальный оператор $G\left(p, t, \xi, D_{\alpha, t}\right)$ для любого действительного в есть ограниченный оператор из $H_{s+m, \alpha}\left(\mathbb{R}_{+}^{1}\right)$ в $H_{s, \alpha}\left(\mathbb{R}_{+}^{1}\right)$.

Доказательство. Из (8) вытекает, что достаточно доказать, что оператор $G\left(p, \tau, \xi, D_{\tau}\right)$, определенный в $(11)$, является ограниченным оператором из $H_{s+m}\left(\mathbb{R}^{1}\right)$ в $H_{s}\left(\mathbb{R}^{1}\right)$.

Обозначим через $\Lambda^{s}\left(p, \xi, D_{\tau}\right)$ псевдодифференциальный оператор вида

$$
\Lambda^{s}\left(p, \xi, D_{\tau}\right) u_{\alpha}(\tau)=F_{\eta \rightarrow \tau}^{-1}\left[(|p|+|\xi|+|\eta|)^{s} F_{\tau \rightarrow \eta}\left[u_{\alpha}(\tau)\right]\right] .
$$

Заметим, что для функции $u(t) \in C_{0}^{\infty}\left(\mathbb{R}_{+}^{1}\right)$ справедливо равенство

$$
\left\|G\left(p, \tau, \xi, D_{\tau}\right) u_{\alpha}(\tau)\right\|_{s}=\left\|\Lambda^{s}\left(p, \xi, D_{\tau}\right) G\left(p, \tau, \xi, D_{\tau}\right) \Lambda^{-m-s}\left(p, \xi, D_{\tau}\right) v(\tau)\right\|_{L_{2}\left(\mathbb{R}^{1}\right)},
$$

где $v(\tau)=\Lambda^{s+m}\left(p, \xi, D_{\tau}\right) u_{\alpha}(\tau),\|\cdot\|_{s}$ - норма в пространстве $H_{s}\left(\mathbb{R}^{1}\right)$. 
Заметим, что $\Lambda^{s}\left(p, \xi, D_{\tau}\right) G\left(p, \tau, \xi, D_{\tau}\right) \Lambda^{-m-s}\left(p, \xi, D_{\tau}\right)$ - псевдодифференциальный оператор с символом из класса $S_{\rho}^{0, p}$. Таким образом, из $(19)$ в силу теоремы 2 получим

$$
\begin{aligned}
\left\|G\left(p, \tau, \xi, D_{\alpha, t}\right) u\right\|_{s, \alpha} & =\left\|G\left(p, \tau, \xi, D_{\tau}\right) u_{\alpha}(\tau)\right\|_{s}= \\
=\left\|\Lambda^{s}\left(p, \xi, D_{\tau}\right) G\left(p, \tau, \xi, D_{\tau}\right) \Lambda^{-m-s}\left(p, \xi, D_{\tau}\right) v(\tau)\right\|_{L_{2}\left(\mathbb{R}^{1}\right)} & \leqslant \\
& \leqslant c\|v(\tau)\|_{L_{2}\left(\mathbb{R}^{1}\right)} \leqslant c\|u(t),|p|\|_{s+m, \alpha}
\end{aligned}
$$

Теорема 3 доказана.

\section{СПИСОК ЛИТЕРАТУРЫ}

1. Баев А. Д. Вырождающиеся эллиптические уравнения высокого порядка и связанные с ними псевдодифференциальные операторы// Докл. АН СССР. - 1982. - 265, № 5. - С. 1044-1046.

2. Баев А. Д. Об общих краевых задачах в полупространстве для вырождающихся эллиптических уравнений высокого порядка// Докл. РАН. - 2008. - 422, № 6. - С. 727-728.

3. Баев А. Д., Бахтина Ж. И., Бунеев С. С., Ковалевский Р. А., Бабайцев А. А. О существовании решений граничных задач в полупространстве для некоторых классов вырождающихся псевдодифференциальных уравнений// Вестн. Воронеж. гос. ун-та. Сер. Физ. Мат. - 2018. - 2. - С. 64-76.

4. Баев А. Д., Бахтина Ж. И., Бунеев С. С., Ковалевский Р. А., Бабайцев А. А. Об априорных оценках решений граничных задач для одного класса вырождающихся псевдодифференциальных уравнений// Вестн. Воронеж. гос. ун-та. Сер. Физ. Мат. - 2018. - 2. - С. 77-92.

5. Баев А. Д., Бахтина Ж. И., Бунеев С. С., Ковалевский Р. А., Бабайцев А. А., Леженина И. Ф., Глушко A. В. Об априорных оценках решений общих граничных задач в полупространстве для вырождающихся эллиптических уравнений// Вестн. Воронеж. гос. ун-та. Сер. Физ. Мат. - 2018. - 3. C. $64-76$.

6. Баев А. Д., Кобълинский П. А. О некоторых свойствах одного класса вырождающихся псевдодифференциальных операторов// Докл. РАН. - 2015. - 460, № 2. - С. 133-135.

7. Баев А. Д., Ковалевский Р. А., Бабайцев А. А., Харченко В. Д. О существовании решений общих граничных задач в полупространстве для вырождающихся эллиптических уравнений// Вестн. Воронеж. гос. ун-та. Сер. Физ. Мат. - 2018. - 4. - С. 51-66.

8. Баев А. Д., Ковалевский Р. А., Кобълинский П. А. О вырождающихся эллиптических уравнениях высокого порядка и псевдодифференциальных операторах с вырождением// Докл. РАН. - 2016. 471, № 4. - С. 387-390.

9. Баев А. Д., Найдюк Ф. О., Бабайчев А. А., Харченко В. Д., Леженина И. Ф., Плетнева О. К. О некоторых начально-краевых задачах для вырождающихся параболических уравнений// Вестн. Воронеж. гос. ун-та. Сер. Физ. Мат. - 2019. - 1. - С. 59-69.

10. Баев А. Д., Панков В. В., Харченко В. Д. Об априорной оценке решений краевой задачи в полосе для вырождающегося эллиптического уравнения высокого порядка// Вестн. Воронеж. гос. ун-та. Сер. Физ. Мат. - 2018. - 4. - С. 161-171.

11. Баев А. Д., Работинская Н. И. О некоторых свойствах одного класса вырождающихся псевдодифференциальных операторов// Докл. РАН. - 2017. - 477, № 1. - С. 7-10.

12. Вишик М. И. Краевые задачи для эллиптических уравнений, вырождающихся на границе области// Мат. сб. - 1954. - 35 (77), № 33. - С. 513-568.

13. Вишик М. И., Грушин В. В. Вырождающиеся эллиптические дифференциальные и псевдодифференциальные операторы// Усп. мат. наук. - 1970. - 25, № 4. - С. 29-56.

14. Глушко В. П. Теоремы разрешимости краевых задач для одного класса вырождающихся эллиптических уравнений высокого порядка// в кн.: Дифференциальные уравнения с частными производными. Тр. семин. акад. С. Л. Соболева. - Новосибирск, 1978. - С. 49-68.

15. Келдыш M. В. О некоторых случаях вырождения уравнений эллиптического типа на границе области // Докл. АН СССР. - 1951. - 77, № 2. - С. 181-183.

16. Левендорский C. З. Краевые задачи в полупространстве для квазиэллиптических псевдодифференциальных операторов, вырождающихся на границе// Мат. сб. - 1980. - 111 (153), № 4. - С. $483-501$. 
17. Михлин С. Г. Вырождающиеся эллиптические уравнения// Вестн. Ленинград. ун-та. - 1954. - 8. C. $19-48$.

18. Олейник О. А. Об уравнениях эллиптического типа, вырождающихся на границе области// Докл. АН CCCP. -1952 . -87 , № 6 . - C. $885-887$.

Баев Александр Дмитриевич

Воронежский государственный университет

E-mail: alexsandrbaev@mail.ru

Бабайцев Андрей Александрович

Воронежский государственный университет

E-mail: 259608@mail.ru

Харченко Виктория Дмитриевна

Воронежский государственный университет

E-mail: dmitrieva9696@gmail.com 